\title{
Wearable Slot Antenna for Biomedical Applications: Mutual Coupling and External Interference
}

\author{
Noor M. ALBADRI, David V. THIEL, Hugo G. ESPINOSA \\ Radio Science Laboratory, School of Engineering and Built Environment, Griffith Univ., Brisbane, QLD 4111, Australia \\ h.espinosa@griffith.edu.au
}

Submitted April 27, 2020 / Accepted May 4, 2020

\begin{abstract}
A small slot antenna has desirable characteristics for radio communications and location of an internal transceiver in-vivo medical applications. The effect of coupling between two identical antennas on the human torso was measured between $2.1 \mathrm{GHz}$ antennas on the skin surface. The effect of an external field was measured as a function of the angle in the horizontal plane to quantify noise isolation. The perimeter separation loss was approximately $0.25 \mathrm{~dB} / \mathrm{mm}$. The external radio source induces currents in the soft conducting tissue resulting in a sinc radiation pattern for the antenna/body combination with a front-to-back ratio of approximately $12 \mathrm{~dB}$. As the UHF band is commonly used in many non-medical applications, there is concern that external radio sources can result in a reduced signal to noise ratio and perturbed field strength measurements on the skin.
\end{abstract}

\section{Keywords}

Wearable slot antenna, electromagnetic interference, noise, mutual coupling, surface fields

\section{Introduction}

A slot in a small conducting box has been suggested as a convenient wearable antenna for measuring the radio field on the skin surface from a subsurface transmitter [1]. This paper addresses two important characteristics of the antenna not yet reported, (a) the mutual coupling between two antennas on the skin surface, and (b) the effect of an in-band radio source on the field strength measurement and communications reliability.

The propagation of electromagnetic signals on and near the surface of anisotropic, lossy media is a challenging problem, particularly when there are multiple layers with different electromagnetic properties. A simple conducting wire at the air/material interface has a complex propagation coefficient related to the electromagnetic properties of the materials on both sides of the boundary. While a closed form solution exists for a plane interface with uniform properties [2], numerical modelling is required for more complex structures [3]. When the transmitter is embedded in the lossy media, then lateral wave propagation effects can be observed on the surface evidenced by significant interference nulls [2], [4]. This interference is caused by the difference in the path attenuation and the time of arrival for the direct ray through the material and a surface wave propagating across the surface. When one half space is layered, then trapped and ducted propagation paths can exist. This effect is commonly observed in clear air propagation caused by temperature and humidity variations in layers close to the earth's surface [5].

The simplest approach for wearable antennas on the skin is to assume that the soft tissue is uniform [6], [7]. Fermat's principle of least time on propagation paths is characterized by the phase constant $\beta$. The radio ray paths with the least travel time have the strongest signal. For lossy media (defined when the conductivity $\sigma$ is not zero), the complex propagation coefficient $\gamma$ is given by [8] as

$$
\gamma^{2}=(\alpha+\mathrm{j} \beta)^{2}=\mathrm{j} \omega \mu \sigma-\varepsilon \mu \omega^{2}
$$

where $\alpha$ is the attenuation coefficient, $\omega$ is the angular frequency of the radiation, and $\varepsilon$ and $\mu$ are the electric permittivity and magnetic permeability of the medium, respectively. The larger the ratio $R$,

$$
R=\frac{\sigma}{\omega \varepsilon}
$$

the higher the conductivity of the medium, the smaller is the skin depth $(\delta=1 / \alpha)$ and the slower the phase velocity $(u)$.

Human soft tissue is inhomogeneous and multi-layered. The electromagnetic properties are different for every layer and every individual. These properties are also frequency dependent. On the torso, the common tissue layers are skin, fat, muscle and the internal organs, and all have different electromagnetic properties [9]. Table 1 outlines some published data on these layers at UHF including the conductivity and permittivity values, their respective $R$ values, skin depth $\delta$ and phase velocity $u$. At UHF frequencies, these parameters lie in the intermediate range between a good conductor and a low loss dielectric so that lateral wave propagation is possible.

The significant difference (5 times the skin depth and $20 \%$ lower phase velocity) between the fat and the other 


\begin{tabular}{|c|c|c|c|c|c|}
\hline $\begin{array}{c}\text { Tissue } \\
\text { type }\end{array}$ & $\begin{array}{c}\boldsymbol{\sigma} \\
(\mathbf{S} / \mathbf{m})\end{array}$ & $\boldsymbol{\varepsilon}_{\mathbf{r}}$ & $\boldsymbol{R}$ & $\boldsymbol{\delta}(\mathbf{m})$ & $\begin{array}{c}\boldsymbol{u} \\
\mathbf{1 0}^{\mathbf{8}} \mathbf{~ m} / \mathbf{s}\end{array}$ \\
\hline skin & 1.46 & 38.0 & 0.33 & 0.022 & 0.486 \\
\hline fat & 0.10 & 5.28 & 0.17 & 0.118 & 1.305 \\
\hline muscle & 1.74 & 52.72 & 0.28 & 0.022 & 0.413 \\
\hline organs & 2.04 & 53.88 & 0.32 & 0.019 & 0.408 \\
\hline
\end{tabular}

Tab. 1. Electromagnetic properties of soft human tissue invivo at UHF (from [9]) with the skin depth and phase velocity.

tissues indicates that ducting of the radio signals in this layer is possible. For every individual, the layers vary in thickness and orientation making numerical modelling a significant challenge.

Wearable antennas have many communications applications in sport, entertainment and in the medical industry as body sensor networks. Since the antenna operates in close proximity or directly attached to the human body, compact design is important for wearer comfort and ease of use [10], [11]. Common requirements of a wearable antenna include a low profile, lightweight, mechanical flexibility, time-saving fabrication, and low cost [12]. The position of the antenna on the body depends on the application. For example:

- The communication channel between the body and an off-body transceiver requires minimal contact and electromagnetic isolation from the skin. This type of remote human monitoring is of interest in sport, military and medical applications [13].

- On-body (OB) communications antennas are directly attached to the human body and operate in close proximity with other body worn or implanted antennas [14], [15].

- In-body (IB) antennas are those imbedded or implanted inside the body. They communicate through the human tissue and are used in medical applications [16].

One example of an on-body antenna is a linearly polarized slot antenna where the slot is pressed against the skin for communications with an internal transmitter [1]. One might assume that this antenna, when tuned for soft tissue contact, would be immune from external sources and other antennas mounted on the body. In this paper we report the performance of this type of antenna on the body. These effects have not been explored in detail previously.

An object in the near field of an antenna becomes part of the radiating structure. If the material is lossy, then the antenna efficiency is reduced. If the object is large, then the radiation pattern has an increased aperture size and so a larger number of side-lobes. Both effects are not well characterized as the human torso is multilayered with skin, fat, muscle and organs.

Given the common use of the $2.45 \mathrm{GHz}$ UHF band for medical applications, as well as for Wi-Fi and Bluetooth communications, the influence of external sources on the total field measured was investigated numerically and experimentally. To avoid stray noise sources, the investiga- tions were conducted at $2.1 \mathrm{GHz}$ using a slightly modified slot antenna tuned for this frequency. This frequency is outside of the internationally defined UHF ISM frequency band.

This paper addresses two important questions:

1. Can the mutual coupling between two antennas on the torso result in interference and inaccurate measurements of the signal level? and

2. What is the effect of external sources on the signal received by a surface mounted, inwardly directed, slot antenna?

Two sets of measurements were made in-vivo on a male participant standing vertically.

\section{Methods and Procedures}

\subsection{Antenna Design}

The inward-looking slot antenna shown in Fig. 1 [1], was reconfigured to resonate at $2.1 \mathrm{GHz}\left(\mathrm{S}_{11}=-24 \mathrm{~dB}\right)$ when pressed against the soft tissue of the human torso invivo. The antenna box was machined aluminum with a resonant slot internally coupled with a monopole probe made from brass sheet. The antenna input impedance was approximately $50 \Omega$ and all measurements were made using a vector network analyzer.

The path loss from an internal radio transmitter can be determined from the line-of-sight path, but with surface propagation also a possibility. If the material is relatively low loss, an interference pattern can be observed on the surface as lateral wave effect [3], [4].

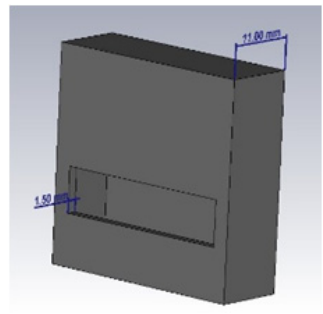

(a)

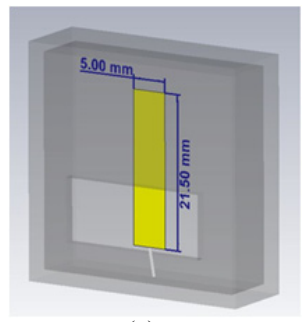

(c)

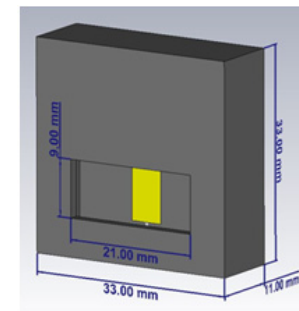

(b)

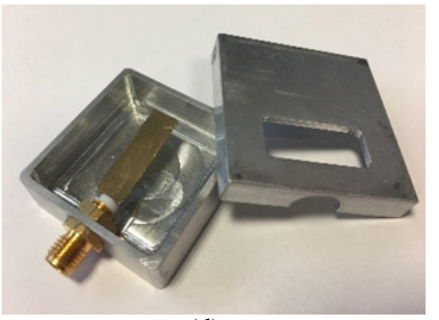

(d)
Fig. 1. The slot antenna consisted of a machined aluminum box with a brass, monopole feed. The antenna was optimized in software to resonate at $2.1 \mathrm{GHz}$ when placed on the human torso: (a-b) box and slot dimensions; (c) monopole dimensions; (d) antenna prototype with a $50 \Omega$ SMA connector. 
The effectiveness of the slot antenna shown in Fig. 1 has previously been reported in on/off-body applications. Some examples include the relationship between the radiofrequency absorption and antenna efficiency at $2.45 \mathrm{GHz}$, for different locations of the antenna on the human body [17], the power absorbed by various body tissues, and the total power absorbed in adult male and female body models [18]. The electric field distribution on the skin surface was measured on multi-layered porcine tissue [19], where two identical slot antennas tuned at $2.45 \mathrm{GHz}$ were pressed against the tissue. The $\mathrm{S}_{21}$ parameter was reported at both epidermal and subdermal locations of the porcine tissue.

\subsection{Measurements and Modelling}

Two antennas were placed directly on human skin (Fig. 2). The measurement was made on the torso of a male participant (age 69 years old, weight $91 \mathrm{~kg}$, height $1.83 \mathrm{~m}$ and BMI $27.2 \mathrm{~kg} / \mathrm{m}^{2}$ ). The measurements were conducted in accordance with Griffith University's ethics research committee approval (GU ref no:2018/601). Table 2 lists the marked locations on the skin with a description and thickness values.

Ultrasound imaging using a GE LOGIQe apparatus, was used to determine the soft tissue thickness values of skin and fat of the trunk at all 7 locations listed in Tab. 2. This provided precise morphological information on the anatomical structure of the participant.

All positions were located under the ribcage and above the iliac crest to avoid scattering from bone. The reflection coefficient $\mathrm{S}_{11}$ was less than $-5 \mathrm{~dB}$ at the resonant frequency of $2.1 \mathrm{GHz}$ for all locations. In Fig. 2, the operator hand holding the antenna in position did not influence the $S_{11}$ measurements nor provided discomfort to the participant.

\subsubsection{Mutual Coupling}

The mutual coupling measurements were recorded at distances between two identical slot antennas. One antenna was located at location 1 for the first measurement, and then at location 2 . The second antenna 2 was placed systematically at the other locations. The two antennas were always parallel and in direct contact with the skin (Fig. 2) and oriented for vertical polarization.

The mutual coupling between the slot antennas was modelled numerically using the electromagnetic FDTD software suite CST Microwave Studio ${ }^{\circledR}$. The multilayer human tissue was modelled as a planar surface using the tabulated electromagnetic properties of human tissue at $2.45 \mathrm{GHz}$ [9]. The body layers included: front surface skin, fat, muscle, soft tissue, fat, back surface skin, and were modelled with the actual thickness obtained from the ultrasound measurements (Fig. 3). The muscle and the inner soft tissue were modelled with a thickness of $100 \mathrm{~mm}$, which was demonstrated to be effectively infinite for $S_{11}$ measurements (skin depth approximately $23 \mathrm{~mm}$ ).

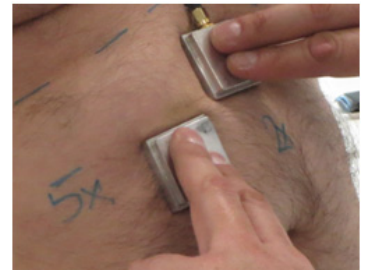

Fig. 2. Antenna 1 and 2 held in direct contact with the skin at position 1 and 4 and set for mutual coupling measurements. The E-field polarization of the antenna is vertical.

\begin{tabular}{|c|c|c|c|}
\hline Location & $\begin{array}{c}\text { Skin } \\
\text { thickness } \\
\text { (mm) }\end{array}$ & $\begin{array}{c}\text { Fat } \\
\text { thickness } \\
\text { (mm) }\end{array}$ & Description \\
\hline 1 & 2.89 & 14.92 & $\begin{array}{c}\text { Locations (1-3) are marked } \\
\text { along the midline of the } \\
\text { abdominal wall, in }\end{array}$ \\
\cline { 1 - 3 } a superior/inferior orientation \\
in line with the umbilicus.
\end{tabular}

Tab. 2. Locations and description of the measurement sites shown in Fig. 2. The layer thickness values were determined from ultrasound measurements on the participant.

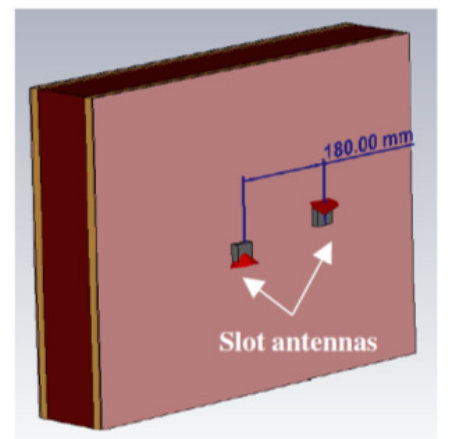

Fig. 3. Numerical modelling configuration. The two slot antennas were positioned in direct contact with the multilayer tissue (front surface skin and back surface skin: pink, fat: yellow, muscle: red, soft tissue: red, fat: yellow). The mutual coupling was determined as a function of separation distance. The structure was changed to mimic the various layer thicknesses at each measurement location.

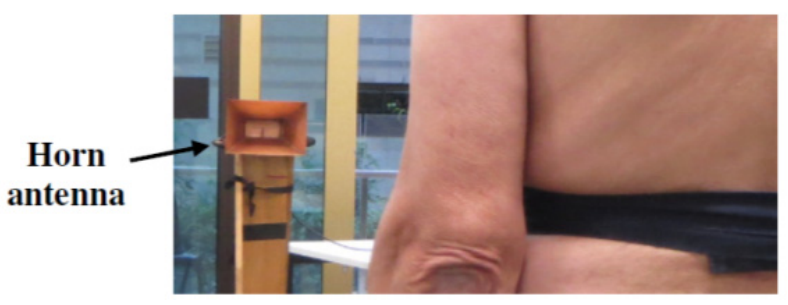

Fig. 4. Vertically polarized horn antenna (the external noise source) directed horizontally to the slot antenna on the front of the torso attached by a Velcro ${ }^{\mathrm{TM}}$ band. 


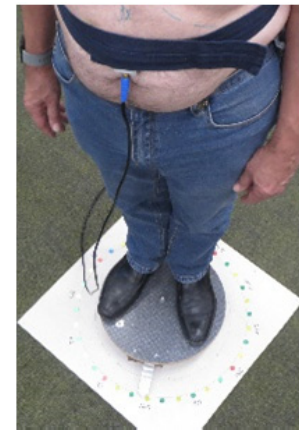

(a)

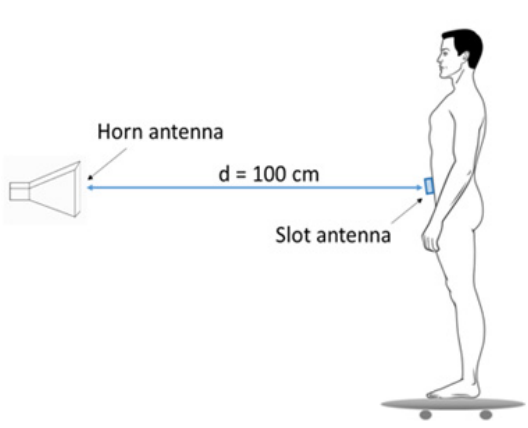

(b)
Fig. 5. (a) The participant standing on the calibrated $360^{\circ}$ rotator with the antenna positioned on the torso. (b) The distance between the horn antenna and the wearable antenna was $100 \mathrm{~cm}$.

\subsubsection{External Interference}

To imitate the effect of an external noise source, a standard gain horn antenna (vertical polarization, center frequency $2.5 \mathrm{GHz}$, aperture size $230 \mathrm{~mm} \times 150 \mathrm{~mm}$ ) was directed towards a slot antenna positioned centrally on the front of the torso (Fig. 4). The antenna was attached at position 2 (Tab. 2) using a Velcro ${ }^{\mathrm{TM}}$ band (this was shown to have no effect on the measurements results).

Two measurement sets were recorded:

(a) The transmission coefficient $\left(\mathrm{S}_{21}\right)$ was recorded at 13 different separation distances $(0.35 \mathrm{~m}-3.41 \mathrm{~m})$.

(b) With the participant standing on a vertical axis, on a $360^{\circ}$ rotator, the $S_{21}$ (horn to slot) was recorded at each angle $\theta$ from $0^{\circ}$ to $360^{\circ}$ in $10^{\circ}$ steps (see Fig. 5(a)). The distance between the wearable antennas to the rotation axis was $100 \mathrm{~cm}$ (Fig. 5(b)).

\section{Results and Discussion}

The mutual coupling simulations and experimental results are shown in Fig. 6. A slight offset in $\mathrm{S}_{21}$ is evident at different circumferential distances between the measured and modelled data. This can be linked to the complex human anatomy and the variations in the dielectric properties of the human tissue not captured in the simulation. This difference is most evident when one antenna is located at position 3. This is thought to be due to the complex tissue structure at this location. The coupling is postulated to be due to partly trapped radiation by the skin layers and so, to some extent, follows the curvature of the torso. The $\mathrm{S}_{21}$ values recorded varied approximately linearly with distance with an attenuation of $-0.25 \mathrm{~dB} / \mathrm{mm}\left(r^{2}=0.79\right)$. The attenuation coefficient is likely to vary between individuals because of anatomical differences (which is not the focus of this paper).

The effect of the external radiation source as a function of distance is shown in Fig. 7. The error bars represent the standard deviation of the received signal at the resonant frequency of $2.1 \mathrm{GHz}$. As expected, the $\mathrm{S}_{21}$ values decreased with increasing separation distance.

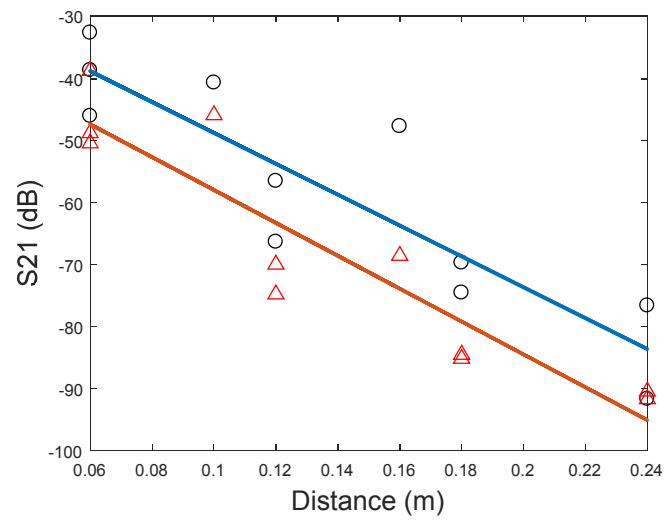

Fig. 6. The $S_{21}(d B)$ at $2.1 \mathrm{GHz}$ showing the mutual coupling variations between the experiment $(\Delta)$ and simulations (o), using two slot antennas located at different separation distances across the skin surface. The fitted straight lines show an approximate linear relationship between $\mathrm{S}_{21}$ and the circumferential distance (Pearson correlation coefficient $r^{2}=0.79$ ).

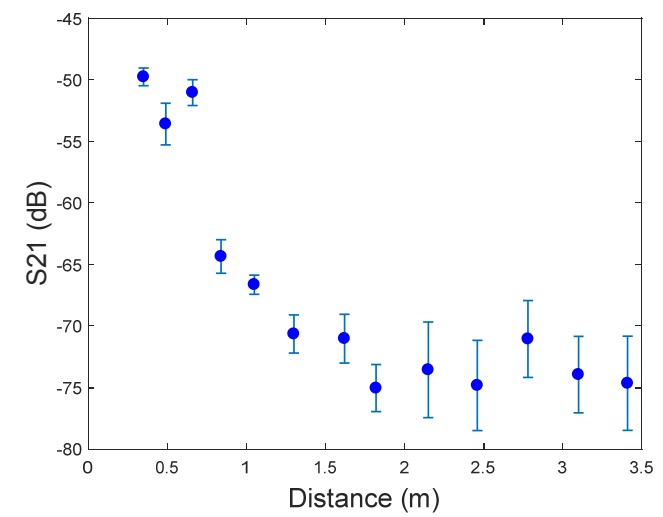

Fig. 7. $S_{21}$ measurements for the horn antenna and the onbody slot antenna as a function of separation distance. The noise level was approximately $-75 \mathrm{~dB}$ at $2.1 \mathrm{GHz}$.

The received signal was recorded as a function of the human rotation angle $\theta$ about the vertical axis, where $\theta=0^{\circ}$ is the front of the human torso (see Fig. 8). While this measurement was not in the far-field zone of the horn, the signal strength has maximum power at the angle $0^{\circ}$ i.e. when antenna on the body is facing the reverse direction. The combination of the human body and the slot antenna results in this principal radiation direction. The radiation pattern $F(\theta)$ of the slot antenna with the human body was fitted to the sinc aperture function [8] common in antenna radiation pattern analysis, but modified to cover the full $360^{\circ}$ as

$$
F(\theta)=\operatorname{sinc}^{2}(0.9 \pi \sin (\theta / 2)) .
$$

While the fit is approximate, the maximum radiation sensitivity lies in the angle range $\pm 90^{\circ}$ and the half power beam-width is approximately $110^{\circ}$. There is also some evidence of a possible lateral wave interference pattern, as minor sidelobes and nulls are present in the measured data. This lateral wave effect was previously demonstrated in field attenuation at $2.4 \mathrm{GHz}$ [20]. The nulls in the theoretical curve cannot match the experimental measurements as the antenna aperture is not infinitely thin. 


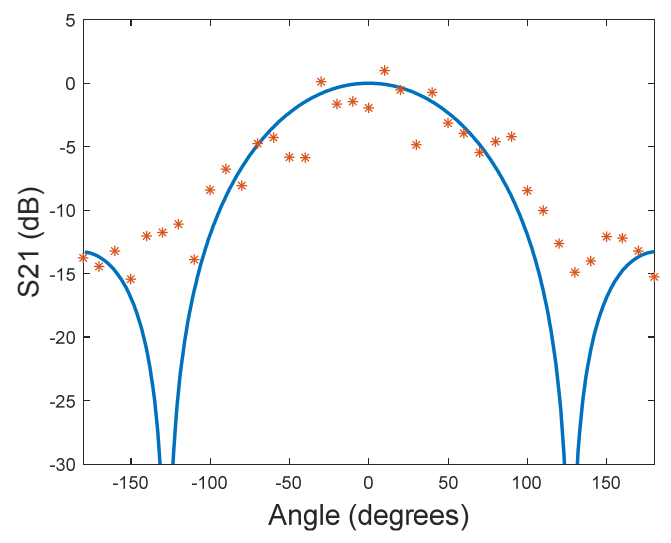

Fig. 8. The normalized radiation pattern of the antenna against the human body at $2.1 \mathrm{GHz}(*)$ with the matched sinc function (continuous line) approximation given in (3).

\section{Conclusion}

An inward-looking, resonant, slot antenna was designed for impedance matching to the human torso at $2.1 \mathrm{GHz}$. Two important characteristics of this antenna were assessed experimentally on a human subject. These were the coupling between two identical slot antennas placed on the soft-tissue region of the torso, and the susceptibility of the antenna to an external noise source. Both measurements are important for determining the location of an ingested transmitter in radio-endoscope measurements. As the passage through the gut can take up to 72 hours, a wearable sensor array is required so that some degree of normal living is possible.

In the current radio environment, the use of UHF signals in the ISM band (Industrial, Scientific and Medical) coincides with both the Wi-Fi and Bluetooth frequencies of radio pill transmitters in common medical use. It is highly likely that a patient will move past Wi-Fi terminals in the normal living environment and likely use a mobile telephone. These external sources, combined with the human movements associated with normal biological functions (cardiac, respiratory, muscular and digestive systems) can impact significantly on the UHF signal quality used for radio pill location.

The major source of external interference occurs when the antenna is closest to the noise source although the slot antenna is inwardly directed. While the front to back ratio of the slot antenna is $>40 \mathrm{~dB}$ in air, when the antenna slot is pressed against the skin, induced currents result in significant field detection from external and adjacent sources. The presence of the fat layer with a lower conductivity was thought to provide a radio duct inside the body resulting in lateral wave propagation and interference in the mutual coupling measurements. The importance of this effect is evident on the human subject used in these measurements and is likely to be particularly important for morbidly obese individuals where the fat layers are much thicker. While the signal levels recorded in these measurements are relatively low, it is clear that the slot antenna is not completely isolated from the environment. It is recommended that during the measurement of signal strength for radio pill location, the nearby UHF sources of the radiation are removed. RSSI measurements based on the coded signal strength can minimize this effect.

Further study into the ducting effect through fat layers might be useful for applications involving intra-torso cavity communications for other biomedical applications.

\section{Acknowledgments}

Noor Albadri would like to thank Griffith University for her Student Postgraduate Scholarship.

\section{References}

[1] FERNANDEZ, M, THIEL, D. V., ARRINDA, A., et al. An inward directed antenna for gastro-intestinal radio pill tracking at 2.45 GHz. Microwave and Optical Technology Letters, 2018, vol. 60, no. 7, p 1644-1649. DOI: 10.1002/MOP/31217

[2] KING, R. W. P., OWENS, M., WU, T. T. Lateral Electromagnetic Waves. New York, (USA): Springer, 1992. ISBN: 9781461391760

[3] KANESAN, M., THIEL, D. V., O'KEEFE, S. G. A robust method of calculating the effective length of a conductive strip on an ungrounded dielectric substrate. Progress In Electromagnetics Research M, 2014, vol. 35, p. 57-66. DOI: 10.2528/PIERM13122404

[4] EMELYANENKO, A., O'KEEFE, S. G., ESPINOSA, H. G., et al. Surface field measurements from a buried UHF transmitter: Theory, modelling and experimental results. IEEE Transactions on Antennas and Propagation, 2017, vol. 65, no. 8, p. 4389-4393. DOI: $10.1109 /$ TAP.2017.2710225

[5] STEPHANSEN, E. T. Clear-air propagation on line-of-sight radio paths: A review. Radio Science, 1981, vol. 16, no. 5, p. 609-629. DOI: 10.1029/RS016i005p00609

[6] CHÁVEZ-SANTIAGO, R., GARCIA-PARDO, C., FORNESLEAL, A., et al. Experimental path loss models for in-body communications within $2.36-2.5 \mathrm{GHz}$. IEEE Journal of Biomedical and Health Informatics, 2015, vol. 19, no. 3, p. 930-937. DOI: 10.1109/JBHI.2015.2418757

[7] SALCHAK, Y. A., ESPINOSA, H. G., THIEL, D. V. Modelling the surface field from an ingested radio transmitter with an approximate attenuation model for gastroenterology investigations. IEEE Transactions on Biomedical Engineering, 2020, vol. 67, no. 2, p. 504-511. DOI: 10.1109/TBME.2019.2916632

[8] ULABY, F. T., MICHIELSSEN, E., RAVAIOLI, U. Fundamentals of Applied Electromagnetics. $6^{\text {th }}$ ed. Boston (Massachusetts, USA): Prentice Hall, 2010. ISBN: 9780132139311

[9] GABRIEL, S., LAU, R., GABRIEL, C. The dielectric properties of biological tissues: II. Measurements in the frequency range $10 \mathrm{~Hz}$ to $20 \mathrm{GHz}$. Physics in Medicine \& Biology, 1996, vol. 41, no. 11, p. 2251-2269. DOI: 10.1088/0031-9155/41/11/002

[10] ARIF, A., ZUBAIR, M., ALI, M., et al. A compact, low-profile fractal antenna for wearable on-body WBAN applications, IEEE Antennas and Wireless Propagation Letters, 2019, vol. 18, no. 5, p. 981-985. DOI: 10.1109/LAWP.2019.2906829 
[11] LIU, Y., LEVITT, A., KARA, C., et al. An improved design of wearable strain sensor based on knitted RFID technology. In IEEE Conference on Antenna Measurements \& Applications (CAMA). Syracuse (NY, USA), 2016, p. 1-4. DOI: 10.1109/CAMA.2016.7815769

[12] AGNEESSENS, S., LEMEY, S., VERVUST, T., et al. Wearable, small, and robust: The circular quarter-mode textile antenna. IEEE Antennas and Wireless Propagation Letters, 2015, vol. 14, p. 1482-1485. DOI: 10.1109/LAWP.2015.2389630

[13] KUMPUNIEMI, T., MÄKELÄ, J. P., HÄMÄLÄINEN, M., et.al. Human body effect on static UWB WBAN off-body radio channels. In 13th EAI International Conference on Body Area Networks (BODYNET). Oulu (Finland), 2018, vol. 2, p. 1-10. DOI: $10.1007 / 978-3-030-29897-5 \_2$

[14] SALONEN, P., RAHMAT-SAMII, Y., KIVIKOSKI, M. Wearable antennas in the vicinity of human body. In IEEE Antennas and Propagation Society Symposium. Monterey (CA, USA), 2004, vol. 1, p. 467-470. DOI: 10.1109/APS.2004.1329675

[15] ABBAS, S. M., ZAHRA, H., HASHMI, R., et al. Compact onbody antennas for wearable communication systems. In International Workshop on Antenna Technology (iWAT). Miami (FL, USA), 2019, p. 65-66. DOI: 10.1109/IWAT.2019.8730638

[16] KIOURTI, A., NIKITA, K. S. A review of in-body biotelemetry devices: Implantables, ingestibles, and injectables. IEEE Transactions on Biomedical Engineering, 2017, vol. 64, no. 7, p. 1422-1430. DOI: 10.1109/TBME.2017.2668612

[17] FERNANDEZ, M., ESPINOSA, H. G., THIEL, D. V., et al. Wearable slot antenna at $2.45 \mathrm{GHz}$ for off-body radiation: Analysis of efficiency, frequency shift, and body absorption. Bioelectromagnetics, 2018, vol. 39, no. 1, p. 25-34. DOI: 10.1002/BEM.22081

[18] FERNANDEZ, M., ESPINOSA, H. G., GUERRA, D., et al. RF energy absorption in human bodies due to wearable antennas in the $2.4 \mathrm{GHz}$ frequency band. Bioelectromagnetics, 2020, vol. 41 no. 1 , p. 73-79. DOI: $10.1002 /$ bem.22229

[19] AlbAdRI, N., SALCHAK, Y. A., ESPINOSA, H. G., et al. E-field distribution in ex-vivo porcine skin layer from a subsurface UHF transmitter. In Proceedings of the $14^{\text {th }}$ European Conference on Antennas and Propagation (EuCAP), 2020.

[20] WANG, L., HU, C., TIAN, L., et al. A novel radio propagation radiation model for location of the capsule in GI tract. In 2009 IEEE International Conference on Robotics and Biomimetics (ROBIO). Guilin (China), 2009, p. 2332-2337. DOI: 10.1109/ROBIO.2009.5420456

\section{About the Authors ...}

Noor M. ABADRI received her M.Sc. in Electrical Engineering from the University of Queensland, Brisbane, Queensland, Australia, in 2016. She is currently working towards the Ph.D. degree at the School of Engineering and Built Environment, Griffith University, Brisbane, Australia. She is working as a sessional instructor in the School of Engineering at Griffith University. Her research interests include wearable antennas, electromagnetic wave propagation, UHF, and in-vivo/ex-vivo measurements.

David V. THIEL received the bachelor's degree in Physics and Applied Mathematics from the University of Adelaide, Adelaide, S.A., Australia, and the M.S. and Ph.D. degrees from James Cook University, Townsville, Qld., Australia. He is currently a Professor in the School of Engineering and Built Environment and Director of the Griffith University Sport Technology (GUST) Laboratories, Griffith University, Brisbane, Qld., Australia. He authored the book Research Methods for Engineers (Cambridge, U.K.: Cambridge Univ. Press, 2014), and coauthored a book on Switched Parasitic Antennas for Cellular Communications (Norwood, MA, USA: Artech House, 2002). He has authored six book chapters, over 150 journal papers, and has coauthored more than nine patent applications. His research interests include electromagnetic geophysics, sensor development, electronics systems design and manufacture, antenna development for wireless sensor networks, environmental sustainability in electronics manufacturing, sports engineering, and mining engineering. Prof. Thiel is a Fellow of the Institution of Engineers, Australia, and a Chartered Professional Engineer in Australia.

Hugo G. ESPINOSA received the bachelor's degree in Electronics and Telecommunications Engineering from the Monterrey Institute of Technology and Higher Education, Mexico, in 1998, the master's degree from the University of Sao Paulo, Sao Paulo, Brazil, in 2002, and the Ph.D. degree (summa cum laude) from the Technical University of Catalonia, Barcelona, Spain, in 2008, both in Electrical Engineering. He has been a visiting researcher at the Federal Polytechnic School of Lausanne, Lausanne, Switzerland, and a Postdoctoral Fellow at the School of Electrical Engineering, Tel Aviv University, Tel Aviv, Israel. Since 2011, he has been with the School of Engineering and Built Environment, Griffith University, Brisbane, QLD, Australia, where he is currently a Lecturer in electronic engineering. His research interests include computational electromagnetics, antennas and propagation, wireless sensor networks, inertial sensors and wearable sensor technology for human monitoring. Dr. Espinosa serves as a Senior Member of the IEEE. 